\title{
Diabetic Foot Ulcer: Synopsis of the Epidemiology and Pathophysiology
}

\author{
Amin Muhammad Ibrahim \\ Department of Endocrinology, University of South Wales, Cardiff, United Kingdom
}

Email address:

ibrahimamin23@yahoo.com

\section{To cite this article:}

Amin Muhammad Ibrahim. Diabetic Foot Ulcer: Synopsis of the Epidemiology and Pathophysiology. International Journal of Diabetes and Endocrinology. Vol. 3, No. 2, 2018, pp. 23-28. doi: 10.11648/j.ijde.20180302.11

Received: May 10, 2018; Accepted: May 29, 2018; Published: June 28, 2018

\begin{abstract}
Over 415 million people had diabetes in 2015 and the number is projected to increase in the coming years. The complications of diabetes affect millions of people all over the world and diabetic foot is one of the most common. The global prevalence of diabetic foot varies significantly and a reasonable proportion of cases end up in amputation. Despite interventions at various levels, it continues to be a menacing issue in the overall management of diabetes. Diabetic foot ulcer (DFU) is classified as neuropathic, ischemic or neuro-ischemic. Neuropathic ulcers originate in the setting of diabetic neuropathy while ischemic ulcers are secondary to vascular disease. Infections and the intrinsic delayed wound healing that characterizes diabetes are important considerations in the pathophysiology of DFU. The prevention of DFU involves adequate glycemic control and modification of risk factors. While health education is a fundamental obligation of health care professionals, it is paramount that patients adopt the guidelines of feet care and integrate them into daily life in trying to prevent diabetic foot and its consequences. The aim of this review article is to summarize the epidemiology, pathophysiology and prevention of diabetic foot ulcer.
\end{abstract}

Keywords: Diabetic Foot Ulcer, Amputation, Neuropathy, Peripheral Arterial Disease

\section{Introduction}

Diabetic foot ulcer is a common problem among people living with diabetes. It is often an unpleasant experience due to its chronicity, unsightly nature and some degree of negative psychological feeling associated with having a chronic wound. Mobility may also become an issue at a point which further compounds the problem. Closely related is the major sequela of limb amputation. Amputation leads to permanent disability with inability to perform some daily activities. As the diabetes epidemic continues to spread, it is logic to anticipate a rise in complications like DFU in the absence of well articulate strategies that are executed at all levels. Hence, particular attention to feet care should be a central focus in educating and managing patients with diabetes to ensure that DFU is either prevented or noticed early enough.

\section{Epidemiology}

The life time risk of DFU in a person living with diabetes is $15 \%$ [1] but it could be up to $25 \%$ [2]. The annual incidence is around $3 \%$ [4]. The geographical variation in the prevalence is related to the prevalence of diabetes as well as sociocultural factors that enhance the occurrence. Also important are the socioeconomic standard and access to quality health care. DFU has been identified as the leading reason for hospitalization among patients with diabetes. It is estimated to account for $25 \%$ of all hospital admissions in patients with diabetes [5]. To illustrate the magnitude of the problem, Walrond ER (2001) reported that $75 \%$ of beds in the surgical ward of Queen Elizabeth hospital in Barbados were at a time occupied by diabetic foot cases [6].

\subsection{Global Prevalence}

A recently published systematic review and meta-analysis by Zhang et. al. (2016) of studies from various continents indicated that Belgium had the highest prevalence globally (16.6\%) [3]. The global prevalence of DFU was $6.3 \%$ and was higher in type 2 diabetes (6.4\%) than type 1 diabetes $(5.5 \%)$. The prevalence in Belgium was followed by Canada (14.8\%) and the United States (13.0\%) [3]. North America had the highest continental prevalence of $13 \%$. Africa was 7.2\%, Asia 5.5\% and Europe 5.1\%. Among European countries the prevalence in Norway was $10.4 \%$, Italy was 
9.7\% and Poland $1.7 \%$. In Asia, India had a prevalence of $11.6 \%$, Thailand $8.8 \%$ and Korea $1.7 \%$. Finally, in Africa the prevalence in Cameroun was $9.9 \%$, Egypt $6.2 \%$, South Africa $5.8 \%$ and Uganda $4 \%$. According to the authors, this study is significant because it is the first time a study will be evaluating the prevalence of DFU on a global scale [3].

A systematic review on the prevalence of DFU in five Arab countries indicated that Saudi Arabia had a mean prevalence of $11.85 \%$ (4.7-19\%), Egypt had 4.2\%(1-7.4\%) and that of Jordan was $4.65 \%(4-5.3 \%)$. Also, the prevalence in Bahrain was 5.9\% and 2.7\% in Iraq [7].

A Report by DanMusa et. al. (2016) from a study conducted in a tertiary hospital in Northwestern Nigeria revealed a DFU prevalence of $6 \%$ which is close to the global average [8]. In the same study $67.2 \%$ of patients were males while $32.8 \%$ were females.

\subsection{Amputations}

Lower extremity amputation (LEA) is the most important consequence of Diabetic foot and it is preceded by an ulcer in $85 \%$ of cases [2]. Diabetes is the leading cause of non traumatic amputation accounting for almost $80 \%$ of cases [9]. Amputation is 10-30 times more likely in people with diabetes than those without the disease [2]. It is estimated that a major amputation is carried out in a person with diabetes somewhere in the world every 30 seconds [2]. Despite the enormous psychological and socioeconomic burden of amputation, patients with LEA are at risk of several problems post amputation e.g. ulceration, infection, repeat amputation and mortality on follow up. This situation calls for more vigilance and diligence in isolating and managing cases of Diabetic foot.

The global incidence of LEA varies from 46.1 to 9600 per $10^{5}$ in people with diabetes compared to $5.8-31$ per $10^{5}$ in those without diabetes. Major LEA was 5.6 to 600 in the population with diabetes [10]. The Caribbean region is noted for a high rate of diabetic foot problems. Hennis et. al. revealed that the overall one year incidence of LEA was 936 per $10^{5}$ in the population with DM. Also women in the region had a disproportionately high amputation rates compared to what is obtainable globally [11]. Hambleton et. al. (2009) reported a mortality rate of 273.9 per 1000 person-years (95\% CI of 207.1-362.3) and 113.4 per 1000 person-years (85.2-150.9) after major and minor amputations respectively. Indeed, all cause mortality rate after diabetes related amputations are very high in the region. The one and five years post amputation survival rates were $69 \%$ and $44 \%$ respectively [12].

In the Nijmegen monitoring project in The Netherlands, the annual incidence of foot ulceration varied between 1.2$3.0 \%$ per year in patients with $\mathrm{T} 2 \mathrm{DM}$ while the annual incidence of LEA was between 0.5 to $0.8 \%$. 10 out of 15 $(66 \%)$ amputees died and $23 \%$ of patients with ulceration had subsequent or previous history of amputation [13].

\subsection{Risk Factors}

a) Gender; evidence from multiple studies have indicated that the risk of DFU is higher in males than in females [3].

b) Age; it is more common in older age groups particularly those above 60 years of age $[1,2]$.

c) Race/Ethnicity; The specific contribution of ethnicity as a risk factor has not been adequately elucidated. Only a few studies have focused on racial predisposition. Leggetter et. al. (2002) evaluated the amputation risk in African Caribbean population and Europeans in the United Kingdom and found that the risk in African Caribbeans was one third that of Europeans [14].

d) Socioeconomic status; Diabetic foot is commoner in lower socioeconomic class. In a study by Adeleye JO (2004), 78.2\% of the diabetic foot patients were from low socioeconomic background [15].

e) Smoking; a well recognized risk factor for macrovascular complications including peripheral arterial disease (PAD).

f) Walking barefooted; this predisposes the feet to injury. A Caribbean study observed that $47 \%$ of patients who had previous ulceration walked barefooted within the house and $17 \%$ walked barefooted outside [16].

g) Poor glycemic control; it worsens neuropathy, vascular disease and delays wound healing. The mean blood glucose in patients with diabetic foot disease was 17.6 $\mathrm{mmol} / \mathrm{L}$ in the study by Adeleye JO [15]

h) Neuropathy; more than half of DFUs are neuropathic in origin [17]

i) Peripheral vascular disease; accounts for about $15 \%$ and $35 \%$, alone and in conjunction with neuropathy [18] respectively.

j) Foot deformity; unequilibrated distribution of pressure in the foot during walking exposes pressure bearing points to ulceration.

k) Previous history of DFU; The underlying pathophysiological abnormalities make patients with previous ulcers prone to developing recurrent DFU.

1) Previous amputation; about half of patients who have had limb amputation subsequently develop ulceration [19].

m)Inappropriate foot wears; tight shoes or shoes that are too loose both predispose the foot to injury.

n) Poor vision; this could be due to diabetic retinopathy with the patient unable to properly identify injurious objects.

o) Trauma; both minor and major trauma could be the origin of a chronic ulcer/wound.

\section{Pathophysiology}

\subsection{Neuropathy}

The definitive mechanism by which diabetes causes neuropathy is yet to be completely understood. However, important clues as to how hyperglycemia causes neuropathy have been unraveled courtesy decades of consistent findings from clinical and animal studies. Credible evidences have identified the polyol pathway as a major culprit in diabetic 
neuropathy. In this pathway which is an alternative to mainstream glycolytic pathway, excess glucose is converted to sorbitol by aldose reductase and the sorbitol is oxidized to fructose by sorbitol dehydrogenase [20]. This pathway is essentially functional in the presence of hyperglycemia and has notable consequences. NADPH and NAD are co-factors in these reactions and the competitive utilization of NADPH jeopardizes the cellular ability to access reduced glutathione which is a potent antioxidant [21]. The reduced level of glutathione translates into the inability to adequately eliminate reactive oxygen species. Hence, oxidative stress ensues which impairs normal nerve function. Also, the accumulation of metabolites leads to reduced synthesis of nerve cell myoinositol which is required for neural transmission [1]. In addition, there is the concept of osmotic cell induced nerve damage which holds that the influx through the polyol pathway leads to nerve cell oedema and dysfunction [22]. This hypothesis has also been advanced as a possible explanation for entrapment neuropathy [23]. Advanced glycation end products and protein kinase $\mathrm{C}$ activation are also thought to contribute to diabetic neuropathy [24].

Sensory, motor and autonomic neuropathies are all involved in the pathogenesis of Diabetic foot. Pain is an unpleasant sensation but it may be all that is required to prevent limb loss in diabetes. Lack of pain sensation exposes the feet of patients with sensory neuropathy to repeated unnoticed trauma. This causes foot injury that is inapparent to the individual until perhaps after a reasonable length of time or when observed by someone else. The resulting wound may become difficult to heal owing to several factors. Also, impaired temperature sensation which is a component of sensory neuropathy can make the instant withdrawal of a foot from hot objects or liquid impossible. This creates an ulcer which may become chronic. Impaired enervation of foot muscles leads to flexionextension imbalance and also muscle atrophy. This causes undue bony prominences and unequal pressure distribution in the foot. Progressively, repeated pressure at focal points within the foot lead to ulceration. Charcot atropathy [2] is the extreme form of foot deformity and it occurs in some diabetic patients. Autonomic neuropathy causes reduced sweating and dryness of the skin. The absence of moisture predisposes the skin to cracks which become potential site for ulceration and portal for bacteria entry.

\subsection{Vascular}

Diabetes is a vascular disease. The integration of this concept into our understanding and approach to diabetes will highly enrich our ability to anticipate and prevent these complications. Peripheral arterial disease which is a macrovascular complication is a known cause of diabetic foot. The endothelial dysfunction that occurs in diabetes leads to reduced production of nitrous oxide (NO) which is a dependable vasodilator and an inhibitor of smooth muscle proliferation [25]. The resultant smooth muscle proliferation contributes to atherosclerosis and vascular narrowing. The vasoconstriction diminishes tissue perfusion.
Also, hyperglycemia promotes increase levels of fibrinogen and plasminogen activator inhibitor which impairs fibrinolysis [26]. Thromboxanes, Von Willebrand factor and factor VIII are equally elevated in diabetes [26]. These and other abnormalities promote platelet adhesion and thrombosis. In addition, the formation of advanced glycation end products which are compounds formed by the non enzymatic reaction between sugars and proteins leads to cross linking of molecules in the extracellular matrix of the basement membrane. This alters the structure of the vessels and promotes stiffness [27]. There is also an increased expression of growth factors and adhesion molecules e.g. Intracellular adhesion molecule-1 (ICAM-1) and Vascular endothelial growth factor (VEGF) [28]. Moreover, hyperlipidemia is common in diabetic patients. Elevated LDL supplies a stream of oxidized LDL which contributes to atherosclerosis [29]. Hypertension also enhances vascular dysfunction in diabetes. The overall effect is that of net reduction in blood supply leading to ischemia and foot ulceration.

\subsection{Delayed Wound Healing}

Factors responsible for the delayed wound healing observed in diabetic foot include vascular disease and neuropathy as discussed above. However, several other molecular factors are equally involved in this phenomenon. Wound angiogenesis is defective in diabetes. In the process of wound healing, a balance is achieved between excess and deficient angiogenesis and this is apparently altered in diabetes [30]. It has been noticed that both excessive and inadequate angiogenesis occur in diabetic complications [30]. In diabetic wound however, there is deficient angiogenesis based on the observation of decreased vascularity and delayed closure time [31]. In addition, defective macrophage function and abnormalities surrounding important players in angiogenesis e.g. pigment epithelium derived factor (PEDF), microRNA (non-coding RNA), platelet derived growth factor (PDGF) e.t.c are suggested mechanisms for delayed wound healing [30].

Matrix Metalloproteinases (MMPs) play an important role in degrading and removal of extracellular matrix [32]. They are also involved in tissue remodeling to ensure a regulated healing process. The activity of these enzymes is noticeably increased in diabetes possibly through the effects of oxidative stress and advanced glycation end products [32]. This increased activity is linked to delayed wound healing.

The inadequate mobilization of bone marrow derived endothelial progenitor cells (EPCs) to the site of injury is another possible mechanism of impaired healing [33]. These cells respond to ischaemia and populate the injury site where they partake in the formation of new vessels. Endothelial nitrous oxide synthase synthesizes NO which is a major stimulus for the migration of EPCs [33]. This process is impaired in diabetes.

\subsection{Infections}

Infection is common in DFUs and contributes to delayed 
wound healing. About 60\% [34] of DFUs have clinically significant infection which could be superficial, deep or osteomyelitis. Although, typical signs and symptoms could be absent; severe infection could present with systemic symptoms e.g. fever, chills and tachycardia. The Infectious Disease Society of America (IDSA) criteria for severe diabetic foot infection are temperature of $>38^{\circ} \mathrm{C}$ or $<36^{\circ} \mathrm{C}$, heart rate $>90 \mathrm{bpm}$, respiratory rate $>20 \mathrm{cpm}$ or $\mathrm{PaCO}_{2}<32 \mathrm{mmHg}$ and white blood cell count $>12000$ or $<4000$ cell count per microliter [35]. Septic wounds often signify a poor prognosis [36]. The commonly isolated organisms are gram positive cocci e.g. staphylococcus aureus, Staph. epidemidis and streptococci species[36]. Methicilin resistant staph aureus (MRSA) complicates 15$32 \%$ [37] of infections and it is associated with treatment failure. Among anaerobes, peptostreptococcus magnus and Bacteriodes fragillis have been isolated. The majority of cases are polymicrobial. Staph. aureus, Group B streptococcus and gram negative bacilli are associated with limb threatening infections[36].

\section{Classification}

Diabetic foot is either neuropathic, ischaemic or neuroischaemic. The common classifacations i.e. Wagner's and University of Texas are based on these parameters in addition to infection and depth of the wound. Other less common classification systems are PEDIS, SINBAD and $\mathrm{S}$ (AD)/SAD [35].

Wagner's Classification

Grade 0- No open lesion

Grade 1- Superficial ulcer

Grade 2- Ulcer extends to the ligament, tendon, joint capsule, deep fascia without abscess or osteomyelitis

Grade 3- Deep ulcer with abscess, osteomyelitis or joint sepsis.

Grade 4- Gangrene localized to portion of the foot or heel

Grade 5- Extensive gangrenous involvement of the entire foot

\section{Prevention of Diabetic Foot Ulcer}

\subsection{Lifestyle Modification}

It is crucial to emphasize that diabetic foot is a diabetic complication. Hence, the lifestyle modifications that are required to prevent or manage diabetes e.g diet and exercise represent an integral component of every strategy necessary to prevent diabetic foot complication. Exercise is one of the most common lifestyle requirements in diabetes and it improves tissue perfusion that is required for wound healing. It is also reported to enhance wound size reduction in DFU [38]. Nevertheless, patients with diabetic foot must seek expert opinion before exercising to avoid potentially deleterious consequences. Smoking cessation is mandatory for high risk individuals. Smoking impairs the function of neutrophils and macrophages which are necessary for proper healing. The effects on wounds include delayed healing, necrosis and promoting infection $[39,40]$.

\subsection{Medications}

Intensive control of blood glucose is required to prevent both macrovascular and microvascular complications. This was abundantly illustrated by UKPDS [41] and DCCT [42] trials. Adequate treatment of hypertension and dyslipidemia is essential to prevent vascular complications.

\subsection{Footcare in Diabetes}

A. Examination and washing: Feet examination is done daily. This is one of the most effective measures in preventing DFU. The entire foot, including the interdigital areas should be inspected by the patient. A relative or caregiver may assist with the feet examination if the patient has visual or mobility problems. Elderly patients could be forgetful and may be required to be reminded or assisted with the feet examination. Feet examination detects any abnormalities e.g. blisters, cuts, cracks e.t.c. The use of mirror has been suggested to provide a better view of all parts of the foot. The feet are washed with soap and water in a gentle manner and dried with a towel. The use of hot water should be avoided. Moisturizers should be applied to prevent dryness and cracks which could be portal for entry of infection. However, the application of creams on the interdigital areas is best avoided [43]. Fungal infections of the feet are common in diabetic patients and should be identified and treated.

$B$. Foot wears; only properly fitting shoes should be used by diabetic patients. Tight shoes do exert pressure on certain parts of the foot and are not recommended. Footwears must fit properly to the size and shape of the feet. Proper inspection of shoes is done before wearing to identify any unwanted object inside. They should be shaken and inverted for any unidentified object to fall out. Well fitting cotton or wool stockings are preferred to nylon stockings because they absorb sweat [43]. Some patients may require custom shoes. These are specially designed footwears which reduce pressure at specific points and conform to the shape of the foot even in the presence of deformity. Footwears are used while indoors and outside. Walking barefooted should be avoided.

C. Nails care; Nails should be well cut with the edges trimmed. They should not be cut too short. In growing toe nails and other nails abnormalities could become a source of injury and should be treated accordingly.

D. Annual feet examination; A comprehensive foot examination is done yearly with a focus on neuropathic, vascular and structural assessment. High risk patients require more frequently examinations.

\subsection{Saint Vincent's Declaration}

In 1989 delegates from European Countries met in St. Vincent, Italy to chart a way forward for the prevention of diabetic foot complications. The major recommendation was to reduce the number of limb amputations for diabetic 
gangrene by $50 \%$ in a five year period [44]. The priority needs were also identified and included:-

a) Regular systematic screening of feet and legs for detection and correction of risk factors.

b) Immediate access to qualified foot care for those at high risk.

c) Systematic patient education in foot care to reduce chronic ulceration and need for surgery.

d) Establishment of multidisciplinary foot care teams, including a podiatrist, nurse, surgeon and physician to provide care for those with complex needs.

\section{Conclusion}

Diabetic foot continues to be a major concern for people living with diabetes despite interventions at different levels to improve the situation. This condition could be ameliorated by maximizing the emphasis on patient education particularly in regards to feet care and intensifying the drive for routine comprehensive feet examination. Patients should be empowered to care better for themselves by getting involved in initiatives that promote awareness of the risk factors and consequences of diabetic foot in addition to how these risk factors can be modified. The establishment of National and local guidelines on feet care should be a priority and the adherence to such guidelines has to be monitored by concerned organizations.

\section{References}

[1] Ismail Lawal Dahiru, Kenneth Ezenwa Amaefule, Innocent Onaja Okpe, Abdulrasheed Ibrahim and Salisu Babura Muazu. An overview of diabetic foot disease. Nigeria Journal of Basic and clinical sciences 2016 Vol. 13 Issue 1Pg 1-12.

[2] M. P. Khanolkar, S. C. Bain, J. W. Stephens. The diabetic foot. QJM Vol. 101, Issue 9, $1^{\text {st }}$ Sep. 2008 Pg 685-695.

[3] Pengzi Zhang, Jing Lu, Yali Jing, Sunyi Yan Tang, Dalong Zhu and Yan Bi. Global epidemiology of diabetic foot ulceration: A systematic review and meta-analysis. Annals of medicine Vol. 49, 2017 Issue 2.

[4] Warren Clayton Jr. and Tom A Elasy. A review of the pathophysiology, classification and treatment of foot ulcers in diabetic patients. Clinical diabetes Vol 27, Number 2009.

[5] David G Armstrong and Lawrence A Lavery. Diabetic foot ulcers: Prevention, diagnosis and classification. American Family physician. March 151998 issue.

[6] Walrond ER. The Caribbean experience with the diabetic foot management of the diabetic foot. West Indian Medical Journal 2001 March 1-4; 50 Suppl 1:24-26.

[7] M. Mairghani, K. Elmusharaf, D. Patton. O Eltahir, G. Jassim and $\mathrm{Z}$ Moore. The prevalence and incidence of diabetic foot ulcer among five countries in the Arab World: A systematic review. Journal of wound care 2017 Sep. 1; 26(supp 9): s27-s24.

[8] Umar Mukhtar DanMusa, Lorliam Terhile, Idris Abdullahi Nasir, Auwal Alkassim Ahmad and Habiba Yahya Muhammad. Prevalence and healthcare cost associated with the management of diabetic foot ulcer in patients attending Ahmadu Bello University Teaching Hospital Nigeria. International Journal of health sciences 2016 April; 10(2):219228.

[9] Trautner C, Haastert B, Giani G, Berger M. Incidence of lower limb amputations and diabetes. Diabetes Care 1996 Sep. 19(9): 1006-9.

[10] Moxey P. W, Gogalniceanu P, Hincliffe RJ, Loftus IM, Jones KJ, Thompson M, Holt PJ. Lower extremity amputation: a review of global incidence. diabetes Medicine 2011 Oct (10):1144-53.

[11] Anselm JN Hennis, Henry S Fraser, Ramesh Jonnalagadda, John Fuller and Nish Charturvedi. Explanation for the high risk of diabetes amputations the Caribbean population of black African descent and potential for prevention. Diabetes Care 2004 Nov; 27(11): 2636-2641.

[12] Ian R Hambleton, Ramesh Jonnalagadda, Christopher $\mathrm{R}$ Davies, Henry S Fraser, Nish Charturverdi and Anslem J Hennis. All cause mortality after diabetes related amputation in Barbados. A prospective study. Diabetes care 2009 Feb; 32(2): 306-307.

[13] Ilona Statius Muller, Wim J. C. de Grauw, Willem H. E. M. van Gerwen, Marie Louise Bartelink, Henk J. M. van den Hoogen and Guy E. H. M. Rutten. Foot Ulceration and Lower Limb Amputation in Type 2 Diabetic Patients in Dutch Primary Health Care. Diabetes Care 2002 Mar; 25(3): 570-574.

[14] Sandra Leggetter, Nish Chaturvedi, John H. Fuller et. al. Ethnicity and Risk of Diabetes-Related Lower Extremity Amputation. A Population-Based, Case-Control Study of African Caribbeans and Europeans in the United Kingdom. Arch Intern Med. 2002; 162 (1):73-78.

[15] Adeleye JO. Diabetic foot disease: the perspective of a Nigerian tertiary health care centre. Volume 22 Issue 6 July/August 2005 Pages 211-214.

[16] Gulliford MC and Mahabir D. Diabetic foot disease and foot care in a Caribbean community. Diabetes Research and Clinical Practice 2002 Apr; 56(1):35-40.

[17] Dyck PJ, Davies JL, Wilson DM, Service FJ, Melton LJ $3^{\text {rd }}$, O'Brien PC. Risk factors for severity of diabetic polyneuropathy: Intensive longitudinal assessment of the Rochester Diabetic Neuropathy Study cohort. Diabetes Care 1999;22:1479-1486.

[18] Maren Volmer-Thole and Ralf Lobmann. Neuropathy and Diabetic Foot Syndrome. International journal of molecular sciences. 2006 17, 917.

[19] Kruse I and Endelman S. Evaluation and treatment of diabetic foot ulcers. Clinical Diabetes 2006; 24:91-3.

[20] Stephen SM Chung, Eric CM Ho, Karen SL Lam and Sookja K Chung. Contribution of the Polyol pathway to Diabetes Induced Oxidative stress. Journal of American Society of Nephrologists Aug. 1 2003. Vol. 14 no. Suppl. 3, s233-236.

[21] Michael Brownlee. The Pathobiology of Diabetic Complications: A unifying mechanism. Diabetes 2005 June 54(6): 1615-1625.

[22] David Tomlinson. Neurobiology of Diabetic Neuropathy. $\begin{array}{lllll}\text { International Review of Neurobiology Vol. } 50 & \text { C2002 }\end{array}$ Elsevier. 
[23] Aszmann OC, Dellon ES, Dellon AL. Anatomical course of the lateral femoral cutaneous nerve and its susceptibility to compression and injury. Plastic and Reconstruction Surgery 1997; 100:600-4.

[24] Feldman EL, Russell JW, Sullivan KA, Golovoy D. New insights into the pathogenesis of diabetic neuropathy. Curr Opin Neurol 1999; 12: 553-63.

[25] American Diabetes Association. Peripheral Arterial Disease in People with Diabetes. Diabetes Care 2003 Dec; 26(12): 33333341.

[26] Aaron I. Vinik, Tomris Erbas, Tae Sun Park, Roger Nolan and Gary L. Pittenger. Platelet Dysfunction in Type 2 Diabetes. Diabetes Care 2001 Aug; 24(8): 1476-1485.

[27] Cooper ME, Bonnet F, Oldfield M and Jandeleit-Dahm K. Mechanism of Diabetic Vasculopathy: An overview. American Journal of Hypertension 2001; 14:475-486.

[28] Guiseppina Basta, Guido Lazzerini, Marika Massaro et. al. Advanced Glycation End-product Activate Endothelium Signal-Transduction Receptor RAGE. A Mechanism for Amplification of Inflammatory Responses. Circulation 2002; $105: 816-822$

[29] Richard Bucala, Zenji Makita, Gloria Vega, Scott Grundy, Theodor Koschinsky, Anthony Cerami and Helen Vlassara. Modification of Low density Lipoprotein by Advanced Glycation End-products Contribute to the Dyslipidemia of Diabetes and Renal Insufficiency. Protocol for the National Academy of Sciences USA. Vol. 91 pp. 9441-9445. Sep. 1994. Medical sciences.

[30] Uzoagu A. Okonkwo and Luisa A. DiPietro. Diabetes and Wound Angiogenesis. International journal of molecular sciences 2017 Jul; 18(7): 1419.

[31] Dinh T and Veves A. Microcirculation of the diabetic foot. Current Pharmaceutical Design. 2005; 11(18):2301-9.

[32] Sandra Matabi Ayuk, Heidi Abrahamse and Nicolette Nadene Houreld. The Role of Matrix Metalloproteinases in Diabetic Wound Healing in relation to Photobiomodulation. Journal of Diabetes Research. 2016; 2016: 2897656.

[33] Harold Brem and Marjana Tomic-Canic. Cellular and molecular basis of wound healing in diabetes. Journal of Clinical Investigation 2007 May 1; 117(5): 1219-1222.

[34] Prompers L, Huijberts M, Apelqvist J, Jude E, Piaggesi A, Bakker K, Edmonds M, Holstein P, Jirkovska A, Mauricio D, Ragnarson Tennvall G, Reike H, Spraul M, Uccioli L, Urbancic V, Van Acker K, van Baal J, van Merode F, Schaper
N. High prevalence of ischaemia, infection and serious comorbidity in patients with diabetic foot disease in Europe. Baseline results from the Eurodiale study. Diabetologia. 2007 Jan; 50(1):18-25.

[35] Benjamin A. Lipsky, Anthony R. Berendt, Paul B. Cornia, James C. Pile, Edgar J. G. Peters, David G. Armstrong, H. Gunner Deery, John M. Embil, Warren S. Joseph, Adolf W. Karchmer, Michael S. Pinzur and Eric Senneville. 2012 Infectious Diseases Society of America Clinical Practice Guidelines for the Diagnosis and Treatment of Diabetic Foot Infections. Clinical Infectious Diseases 2012; 54(12):132-173.

[36] Kimberlee B. Hobizal and Dane K. Wukich. Diabetic foot infections: current concept review. Diabetic Foot Ankle. 2012 Vol. 3 Issue 1.

[37] Eleftheriadou I, Tentolouris N, Argiana V, Jude E, Boulton AJ. Methicillin-resistant Staphylococcus aureus in diabetic foot infections. Drugs. 2010 Oct 1; 70(14):1785-97.

[38] Eraydin Ş and Avşar G. The Effect of Foot Exercises on Wound Healing in Type 2 Diabetic Patients with a Foot Ulcer. Journal of Wound, Ostomy and Continence Nursing. 2017 Dec 19.

[39] Jodi C. McDaniel. Smoking, Chronic Wound Healing, and Implications for Evidence-Based Practice Journal of Wound Ostomy and Continence Nursing. 2014 Sep-Oct; 41(5): 415E2.

[40] Min Liu, Wei Zhang, Zhaoli Yan, and Xiangzhen Yuan. Smoking increases the risk of diabetic foot amputation: A meta-analysis. Experimental and Therapeutic Medicine 2018 Feb; 15(2): 1680-1685.

[41] American Diabetes Association. Implications of the United Kingdom Prospective Diabetes Study. Diabetes Care 2002 Jan; 25(suppl. 1): s28-s32.

[42] American Diabetes Association. Implications of the Diabetes Control and Complications Trial. Diabetes Care 2003 Jan; 26 (suppl. 1): s25-s27.

[43] C. Keith Bowering. Diabetic foot ulcers: Pathophysiology, assessment, and therapy. Canadian Family Physician 2001; 47:1007-1016.

[44] Preventing foot complications in diabetes: the St Vincent Declaration 26 years on. PRACTICAL DIABETES VOL. 33 NO. 5 Copyright (C) 2016 JOHN WILEY \& SONS. http://www.practicaldiabetes.com/ accessed 22/4/2018 